Atmos. Res., Boulder, Colo., U.S.A..

Whang, Y. C., and Burlaga, L. F.: 1988, Evolution of recurrent solar wind structures between 14 AU and the termination shock, J. Geophys. Res., 99, 5446-5460.

Winske, D.: 1988, Numerical modeling of slow shocks, Proc. of the Sixth Internatl. Solar Wind Conf. (V. J. Pizzo, T. E. Holzer, and D. G. Sime, eds.), NCAR/Tech. Note 306+Proc, 387-400, Natl. Center for Atmos. Res., Boulder, Colo., U.S.A.

Winterhalter, D., Smith, E. J., and Slavin, J. A.: 1988, The radial gradient in the interplanetary magnetic field between $1 \mathrm{AU}$ and $23 \mathrm{AU}$, Proc. of the Sixth Internatl. Solar Wind Conf. (V. J. Pizzo, T. E. Holzer, and D. G. Sime, eds.), NCAR/Tech. Note 306+Proc, 587-592, Natl. Center for Atmos. Res., Boulder, Colo., U.S.A..

Withbroe, G. L.: 1988, The temperature structure, mass, and energy flow in the corona and inner solar wind, Astrophys. J., 925, 442-467.

Wu, S. T., Panitchob, S., and Suess, S. T.: 1988, Adaptive grid MHD simulation of solar disturbances propagating from the Sun to $1 \mathrm{AU}$, Proc. of the Sirth Internatl. Solar Wind Conf. (V. J. Pizzo, T. E. Holzer, and D. G. Sime, eds.), NCAR/Tech. Note 306+Proc, 303-307, Natl. Center for Atmos. Res., Boulder, Colo., U.S.A..

\title{
Plasma Processes in the Heliosphere
}

Frank Verheest

Rijksuniversiteit Gent, Krijgslaan 281, B-9000 Gent, Belgium

In this review a few plasma processes in the heliosphere are highlighted. Undoubtedly the major event from mid-1987 to mid-1990 was the Voyager 2 flyby of Neptune on 25 August 1989, of which results are now becoming available. However, most papers dealt with the processing and interpretation of the results from the flyby of Uranus (on 24 January 1986), as well as from earlier encounters with Jupiter and Saturn. Similarly, there continued to be great interest in results from the spacecraft missions to comets $\mathrm{P} /$ Giacobini-Zinner and P/Halley in 1985-1986. Mention should also be made of the Phobos missions to Mars and its satellites which yielded valuable scientific results, though unfortunately prematurely aborted. Of necessity, only recent literature will be cited, as these references include earlier ones.

\section{PLASMA WAVES AND INSTABILITIES IN COMETARY ENVIRONMENTS}

Most of the work of interest deals with the pickup by the solar wind (SW) of newly ionized particles of cometary origin, and the influence of this on the SW flow around the nucleus, with the creation of a bow shock. Volatile material (mostly water ice) is outgassed from the nucleus and escapes from the collision-dominated inner coma, unhampered by the nucleus' low gravity. Subsequent ionization of these neutral particles by photoionization, charge exchange with SW ions and electron impact (Cravens et al. 1987; Gombosi 1988) occurs on a slow timescale, allowing some particles to reach distances of $8 \times 10^{6} \mathrm{~km}$ from the nucleus (Neugebauer et al. 1987).

The distribution of new cometary ions contains essentially two sources of free energy, beam-like velocity differences with the SW parallel to the interplanetary magnetic field (IMF) and a perpendicular gyrating ring distribution in velocity space. Both sources generate low-frequency magnetohydrodynamic (MHD) turbulence, resulting in fast pitch angle scattering of the new ions into a shell distribution in the SW frame, accompanied by a slower diffusion in energy (Price $\&$ Wu 1987; Gaffey, Winske $\otimes W u$ 1988; Coates et al. 1989,1990). This is similar to, but much stronger than the pickup by the SW of interstellar hydrogen and helium (Lee \& Ip 1987; Fahr \& Ziemkiewicz 1988; Möbius et al. 1988) or of charged interstellar dust (Wallis 1987). General discussions of the different aspects of the role of Alfvén waves in cometary environments have been given, from a more theoretical (Galeev 1987,1989; Galeev 8 Sagdeev 1988; M.A. Lee 1989) as well as from an observational point of view (Scarf 1989).

Inducing new or amplifying intrinsic SW MHD turbulence occurs mostly due to resonant instabilities, corresponding to fluctuating magnetic field energy spectra with clear peaks at the oxygen ion gyrofrequency (Thorne \& Tsurutani 1987; Yumoto, Saito \& Nakagawa 1987; Price \& Lee 1988; Gary, Akimoto \& Winske 1989) or its harmonics (Goldstein, Wong \& Glassmeier 1990). Coexisting cometary proton and oxygen bearns excite resonant instabilities without undue influence of other species (Brinca \& Tsurutani 1988; Gary et al. 1988). Instabilities with right-hand polarization in the zero momentum frame have larger linear growth rates (Gary \& Madland 1988). Both Alfvén and magnetosonic wayes grow preferentially parallel to the IMF, with growth rates increasing with both the newborn ion density and the angle between the IMF and the SW (Price, Gaffey $\&$ Dong 1988). However, some models predict sizeable growth rates also for oblique modes (Brince \& Tsurutani 1989; Wu \& Yoon 1990; Yoon 1990).

Under certain plasma conditions one would also expect nonresonant beam instabilities (Lakhina 8 Verheest 1988; Price 1989), although their long wavelengths would make these hard to detect, and there has been no unambiguous confirmation at comets sofar. Nevertheless, stochastic acceleration theories would require such waves in order to get cometary ions to the observed energies (Galeev et al. 1990).

Besides the already mentioned low-frequency turbulence, there is evidence that cometary ions, in particular protons, can also excite whistlers and waves with frequencies much higher than the proton gyrofrequency but with mixed polarization ( $W u, H e \&$ Price 1988). Sometimes, these whistler modes are generated at the leading edge of steepened low-frequency waves or shocklets (Tourutani et al. 1987; Le, Russell \& Smith 1989), as also observed upstream of Earth's bow shock (Wong Goldstein 1988).

Finally, there seems to be some evidence that solitary magnetic, non-compressional transverse pulses have been observed during the ICE mission to P/Giacobini-Zinner (Tsurutani et al. 1989). Nonlinear interaction between Alfvén solitons and Langmuir waves could explain the cometary kilometric radiation observed near $\mathrm{P} /$ Halley (Lakhina $\&$ Buti 1988), while Alfvén solitons might be relevant in explaining the properties of MHD turbulence at comets (Lakhine $\&$ Shukle 1987; Verheest 1990) and in the 
heliosphere in general (Hada, Kennel \& Buti 1989; Ponce Dawson \& Ferro Fontán 1990).

For the cometary ion pickup process the agreement between theoretical explanations, numerical simulations and observations is very good. While cometary environments suggested the model parameters here, similar conclusions hold for observations of low-frequency wave activity elsewhere in space, inside and outside the heliosphere. Some examples among many are the ion pickup at Venus (Breus, Krymskii 8 Mitnitskii 1987) and the generation of low-frequency noise in Earth's plasma sheet boundary layer by electromagnetic beam instabilities (Angelopoulos et al. 1989; Gary $\&$ Winske 1990).

The cometary mass loading of the SW is also responsible for the creation of the cometary bow shock, ionopause and associated boundary layers (Neubauer 1987,1988; Mendis et al. 1989), although the precise formation process is still very disputed. For planets with intrinsic magnetic fields the thickness of the interaction region between the SW and the magnetosphere is small. Unmagnetized comets, however, do not really present a rigid obstacle to generate a shock, and this creates a wide interaction region of the size of the coma. As the SW possesses an intrinsic dispersive length scale, the interplay between these two very different scales could determine the bow shock in the case of comets, by supporting quasi-stationary solitons (Khabibrakhmanov \& Verheest 1990). A supermagnetosonic to submagnetosonic transition can thus be accomplished in a mass loaded SW (Hizanides, Cargill \& Papadopoulos 1988; Sauer, Motschmann $\&$ Roatsch 1990). Interpreting steepened waves as shocks would suggest that $\mathrm{P} /$ Giacobini-Zinner did not have a single bow shock but a transition region consisting of a series of shocks convected by the SW (Omidi \& Winske 1988).

\section{PLASMA PROCESSES AT URANUS AND NEPTUNE}

The Voyager missions learned us that the four giant gas planets all possess intrinsic magnetic fields, and hence magnetospheres with trapped radiation belts and plasma wave turbulence. While Saturn's magnetic field may adequately be represented by a dipole aligned with the rotation axis but with a small offset to the north, Jupiter's magnetic field is tilted by about $10^{\circ}$ from the rotation axis. Moreover, electric currents along field lines connecting Jupiter and Io and in a current sheet around Jupiter's equator distort the field sufficiently so as to make a simple offset dipole field a poor representation. On the other hand, both Uranus and Neptune have magnetic fields which are highly tilted with respect to their rotation axes and offset from their centres by large fractions of the planetary radii.

Due to the $60^{\circ}$ tilt of the Uranian dipole field, Voyager 2 was able to explore a large range of magnetic latitudes and find a marked inbound-outbound asymmetry. Uranus' nonthermal radio emissions are rather weak compared to those of Jupiter and Saturn, but comparable in complexity, with as many as six distinctly different types. Both smooth and bursty components were observed. General reviews have been given, dealing also with other planets (Anderson \& Kurth 1989; Kaiser 1989; L.C. Lee 1989).

Smooth low-frequency radio components $(20-347 \mathrm{kHz})$ are left-hand/right-hand polarized when observed in the northern/southern magnetic hemisphere, without polarization reversal from dayside to nightside, while high-frequency emissions (up to $865 \mathrm{kHz}$ ) are only observed in the nightside and with lefthand polarization. The bursty emissions are sometimes broadband $(78-750 \mathrm{kHz})$, sometimes narrow-band $(40-270 \mathrm{kHz}$ ) (Leblanc et al. 1987). Intense plasma wave activity occurs only in the inner magnetosphere $\left(r<12 R_{U}\right)$, similar to the situation at Saturn, but in marked contrast with Jupiter, where very strong wave activity was detected out to distances of $250 R_{J}$ (Scarf et al. 1987). Finally, there is a weak continuum radiation at $1-3 \mathrm{kHz}$, more like that detected at Saturn than the extremely intense Jovian continuum. The low intensity of the Uranian continuum is attributed to the lack of a density cavity in the magnetosphere, which is deep enough relative to the SW (Kurth, Gurnett \& Desch 1990).

The smooth low-frequency kilometric radiation is ascribed to sources radiating near the local electron gyrofrequency in the extraordinary mode, confined to the magnetic equatorial plane and encircling the planet at distances of a couple of radii (Lecacheux \& Ortega-Molina 1987; Kaiser, Desch \& Connerney 1989), while the higher-frequency radiation is attributed to sources on a small set of field lines whose foot points lie near the magnetic southern (dark) pole of Uranus (Kaiser, Desch \& Curtis 1987; Barbosa 1988). The right-hand polarized, narrow-band bursty signals are due to extraordinary magnetoionic modes emitted in the vicinity of the north (weak) magnetic pole (Desch, Kaiser $\&$ Kurth 1989 ) or to nonlinear interaction between whistler solitons and upper-hybrid waves, caused by energetic electrons on auroral field lines (Buti \& Lakhina 1988). 
In addition to radio emissions, there are electrostatic Bernstein modes between harmonics of the electron gyrofrequency (Kurth et al. 1987). Large-amplitude, very low-frequency (20-40 $\mathrm{mHz}$ and $100-$ $400 \mathrm{mHz}$ ) magnetic field waves have been observed upstream of the Uranian bow shock, simultaneously with energetic charged particles, suggesting that these waves are obliquely propagating whistler modes driven unstable by gyrating proton populations (Smith, Goldstein $\mathcal{O}$ Wong 1989). Such a turbulence is due to the increase of the magnetosonic Mach number with increasing heliospheric distances, as well as to a weaker than expected magnetic field upstream of the bow shock (Russell, Lepping $\theta S$ mith 1990).

Because Uranus and Neptune are rather similar examples of oblique rotators with large magnetic field tilts and offsets, the preliminary picture of plasma wave phenomena at Neptune closely parallels what was said in the above paragraphs about Uranus, even though in the case of Neptune Voyager 2 observed for the first time a planetary magnetic field nearly pole-on to the Sun (Kurth 1990). In particular, the observations included radio emissions, electron plasma oscillations in the SW upstream of the bow shock, electrostatic turbulence at the bow shock, chorus, hiss, electron cyclotron waves and upper hybrid resonance waves in the inner magnetosphere (Gurnett et al. 1990). Also, speculation about the generation of a plasma torus in Neptune's magnetosphere by its satellite Triton (Delitsky, Eviatar \& Richardson 1989) proved correct, much as occurs at Jupiter with the Io plasma torus (Ip 1990). In contrast, Voyager 2 did not detect any heavy ion plasma at Uranus, despite the presence of several large, icy moons within the Uranian magnetosphere (Cheng 1987).

\section{PLASMA PROCESSES AT OTHER PLANETS}

Jovian decametric radio emissions (observable also from Earth) occur as relatively smooth or else very brief, intense and much burstier radiation. The bursts and some of the smooth emissions are observed when the Jovian moon Io occupies specific geometric configurations, implying that such emissions at the local electron gyrofrequency originate on or near magnetic field lines which thread through Io (Genova \& Calvert 1988). In particular the manner in which Alfvén waves interact strongly with the Io plasma torus and magnetic field inhomogeneities is essential in understanding the Voyager observations (Wright 1987; Bagenal \& Leblanc 1988; Glassmeier et al. 1989).

The narrow-band kilometric radiation observed by Voyager is explained by nonlinear coupling between upper-hybrid electrostatic plasma waves in the Io plasma torus (Fung 8 Papadopoulos 1987) or by localized nonlinear vortex structures due to radial plasma gradients in rotating plasmas (Horton 8 $S$ mith 1988). Other electrostatic waves are identified as hydrogen-oxygen Buchsbaum modes, hydrogen Bernstein modes and lower-hybrid waves, all excited by ion pickup in the Io torus (Barbosa 8 Kurth 1990). Finally, low-frequency $(\sim 10 \mathrm{kHz})$ radio bursts bear a striking similarity to solar III type radio bursts and suggest a similar source mechanism, involving energetic electrons moving through a density gradient in the Io plasma torus, as the periodicity of these bursts is similar to the Alfvén wave travel time from one hemisphere to the other through the lo torus (Kurth, Gurnett $\&$ Scarf 1989).

At the quasi-perpendicular supercritical bow shock (see below) of Saturn, plasma waves can generate a significant portion of the total electron temperature jump measured across the shock. In this respect, Saturn's bow shock more closely resembles Jupiter's than Earth's, where plasma waves do not contribute or only negligibly to the electron temperature jump (Moses et al. 1988).

Mars' interaction with the SW may be like Venus', that of a supermagnetosonic plasma flowing past an unmagnetized body with an ionosphere. However, at Mars the SW pressure usually exceeds the ionospheric plasma pressure, while at Venus this condition prevails only for extraordinarily high incident dynamic pressures (Luhmann et al. 1987; Breus et al. 1989). Thus at Mars the weak ionospheric magnetic field is mostly induced, as confirmed by Phobos 2 (Yeroshenko et al. 1990).

Further observations by Phobos 2 suggest that a torus/ring resides along the orbit of the Martian satellite Phobos, interacting strongly with the $\mathrm{SW}$, much as happens near a comet (Dubinin et al. 1990; Ip 8 Banaszkiewicz 1990). Measurements reveal a turbulent bow shock with a strong foot, consistent with reflection of SW protons (Schwingenschuh et al. 1990). High-frequency ion-acoustic waves are excited at the shock front due to currents flowing along the front and then convected inside by the SW. Lowfrequency waves close to the inside boundary are believed to be generated by the diffusion of Martian heavy ions through the planetopause into the magnetosheath (Sagdeev et al. 1990) or by the pickup of protons from the Martian hydrogen exosphere (Russell et al. 1990). 


\section{INTERPLANETARY SHOCKS AND EARTH'S BOW SHOCK}

Shocks in the heliosphere include planetary and possibly also cometary bow shocks, corotating shocks produced by the interaction of high- and low-velocity SW streams and interplanetary travelling shocks produced in the course of coronal mass ejections. The angle between the upstream magnetic field and the shock normal is of critical importance for the shock structure. Whereas at quasi-perpendicular shocks the plasma parameters change abruptly at a thin shock front, the jumps at a quasi-parallel shock occur in a much broader region (Terasawa $\&$ Scholer 1989).

Recent theoretical models of high Mach number quasi-perpendicular shocks predict for both the bulk plasma parameters and the magnetic field an overshoot followed by damped oscillations, as in the Uranian bow shock (Bagenal et al. 1987). In the discussion about planetary foreshocks (upstream of the bow shock) one important point concerns the role played by the shock structure in controlling the MHD upstream turbulence. Large-amplitude, low-frequency waves detected by Voyager 2 in association with the quasi-perpendicular structure of the Jovian bow shock prove that upstream turbulence is not really a characteristic signature of quasi-parallel shocks (Bavassano-Cattaneo et al. 1987).

Slow-mode shocks can arise from the steepening of linear slow-mode MHD waves and might form ahead of objects moving through a magnetized plasma at speeds greater than the slow-mode speed (Wolfson 1987), but are most probable in the slow SW near and at corotating regions (Richter $\&$ Marsch 1988). The decrease in Alfvén speed at increasing heliocentric distances causes a forward slow shock to evolve into a forward fast shock, accompanied during the transition by a rotational discontinuity (Whang 1988). The structure of MHD fast and slow shocks depends upon the relative importance of the different dissipation mechanisms. By ordering the lengthscales for resistivity, thermal conduction and viscosity, one can define upper limit Mach numbers for both fast and slow shocks, at which resistivity and thermal conduction together provide all the shock dissipation without need for viscosity (Kennel 1988).

Hot diamagnetic cavities have sometimes been observed upstream of Earth's bow shock near transitions between quasi-perpendicular and quasi-parallel shock geometries, in close relation with intervals of dense, nearly specularly reflected ions which create the cavity and launch outgoing Alfvén waves (Thomsen et al. 1988; Thomas \& Brecht 1988). Similarly, large-amplitude magnetic pulsations observed upstream of quasi-parallel portions of Earth's bow shock may be a another manifestation of the same basic processes, namely the coupling of coherently reflected ions to the SW beam (Onsager et al. 1990; Thomsen et al. 1990). In the higher energy range (above $\sim 300 \mathrm{keV}$ ) energetic ions upstream of Earth's bow shock are of magnetospheric origin, whereas the ISEE mission has accumulated evidence that in the lower energy range $(\leq 200 \mathrm{keV})$ the bow shock itself is an important source of upstream ions: SW ions are either directly reflected at the quasi-perpendicular shock portions or accelerated by a first-order Fermi mechanism at the quasi-parallel shock portions (Scholer et al. 1989). Also, diffuse ion populations occurring upstream of Earth's bow shock when the IMF was nearly radial have been attributed to reflected SW ions rather than leaked magnetospheric ions (Gosling et al. 1989).

\section{WAVES AND TURBULENCE IN THE SOLAR WIND}

Large-amplitude Alfvén waves as well as MHD discontinuities have long been observed in the interplanetary medium, which thus can be regarded as a natural plasma laboratory to test not only particle acceleration models but also nonlinear theories of finite-amplitude waves, in association with interplanetary shocks, planetary bow shocks and cometary environments (Hada, Kennel \& Buti 1989; Terasawa 8 Scholer 1989), as touched upon already in the preceding sections.

Two complementary views of the origin of SW magnetic and velocity fluctuations hold that the SW turbulence is either driven by velocity shear or else a remnant of solar-generated, outward propagating and decaying waves (at least inside $1 \mathrm{AU}$ ). In the inner solar system, most of the waves, shocks and rotational discontinuities propagate outward from the Sun, the more so when the $S W$ velocity is higher, indicating indeed generation by the Sun. From Helios data between 0.3 and $1 \mathrm{AU}$ one finds that damping of Alfvén waves and heating of SW protons occur simultaneously ( $T u$ 1988). The highest degree of Alfvénicity is found in fast streams near the Sun or in regions with a high degree of incompressibility, suggesting there local generation of turbulence at a velocity shear (Bavassano \& Bruno 1989; Bruno et al. 1989). Moreover, inward propagating Alfvén modes, even if a minority component of the inner solar system turbulence, are nevertheless important and in order to be present in interplanetary space have to be generated beyond the Alfvénic critical point (Bavassano \& Bruno 1989), but seldom steepen into discontinuities before reaching $1 \mathrm{AU}$ (Neugebauer \& Buti 1990). 
Similar conclusions follow from a study of interplanetary MHD fluctuations and the Alfvénicity of SW fluctuations, using Voyager data between 1 and $11 \mathrm{AU}$. It shows again that the SW cannot be viewed as merely a superposition of large-amplitude waves, as these undergo substantial dynamical evolution at all heliocentric distances examined, but that fluctuations must indeed be produced outside the Alfvénic critical point (Roberts et al. 1987). Furthermore, by decomposing the turbulent energy into outgoing and inward components, one finds that Alfvénic periods, where the energy is dominated by outgoing components, occur mainly when density fluctuations are low and the temperature is high (Grappin, Mangeney \& Marsch 1990). Such a decomposition in outgoing and inward components also suggests that isotropic turbulence with a Kolmogorov index of $5 / 3$ is the ultimate state towards which SW MHD fluctuations evolve (Marsch \& Tu 1990; Tu, Marsch \& Rosenbauer 1990).

It would seem that the power level of heliospheric fluctuations in the inner heliosphere is not sufficient to accelerate high-speed streams (Roberts, Goldstein \& Klein 1990), but SW proton double streams and proton-alpha differential streaming may provide sufficient free energy to drive field-aligned magnetosonic waves unstable. Alpha particles by themselves are not able to excite these waves but mostly tend to stabilize existing proton double streams by enhancing the gyrodamping of the main proton population (Marsch \& Livi 1987).

\section{FINAL REMARKS}

In view of the many different occurrences in heliospheric plasmas of strong interactions between energetic particles and large-amplitude waves, there is a need for proper theoretical investigations of the truly nonlinear regimes, which sofar have mostly been explored via numerical simulations and rather ad hoc quasi-linear theories. As is now also emerging, the rôle of chaotic phenomena in the heliosphere has barely been addressed.

\section{REFERENCES}

(COSPAR = Abstracts of the XXVIII Plenary COSPAR Meeting, The Hague, the Netherlands, 25 June to 6 July 1990; GM59 = "Plasma Waves and Instabilities at Comets and in Magnetospheres", Geophysical Monograph 53, ed. by B.T. Tsurutani \& H. Oya, American Geophysical Union, Washington $D C, 1989 ; G R L=$ Geophysical Research Letters; JGR = Journal of Geophysical Research)

Anderson R.R. \& Kurth W.S.: 1989, GM59, p $81 \quad$ - Angelopoulos V., Elphic R.C., Gary S.P. \& Huang C.Y.: 1989, JGR 94, p 15373 - Bagenal F., Belcher J.W., Sittler E.C.,Jr \& Lepping R.P.: 1987, JGR 92, 8603 - Bagenal F. \& Leblanc Y.: 1988, Astron. Astrophys. 197, p 311 • Barbosa D.D.: 1988, Astrophys. J. 333, p 443 - Barbosa D.D. \& Kurth W.S.: 1990, JGR 95, p 6443 - Bavassano B. \& Bruno R.: 1989, JGR 94, p 168 \& p 11977 - Bavassano-Cattaneo M.B., Moreno G., Scotto M.T. \& Acuña M.: 1987, JGR 92, p 12413 • Breus T.K., Krymskii A.M. \& Mitnitskii V.Ya.: 1987, Planet. Space Sci. 35, p 1221 • Breus T.K., Bauer S.J., Krymskii A.M. \& Mitnitskii V.Ya.: 1989, JGR 94, p 2375 - Brinca A.L. \& Tsurutani B.T.: 1988, JGR 93, p 48 - Brinca A.L. \& Tsurutani B.T.: 1989, JGR 94, p 3 \& p 5467 • Bruno R., Bavassano B., Rosenbauer H. \& Mariani F.: 1989, Adv. Space Res. 9, p (4)131 - Buti B. \& Lakhina G.S.: 1988, GRL 15, p 1149 • Cheng A.F.: 1987, JGR 92, p 15309 - Coates A.J., Johnstone A.D., Wilken B., Jockers K. \& Glassmeier K.-H.: 1989, JGR 94, p 9983 - Coates A.J., Wilken B., Johnstone A.D., Jockers K., Glassmeier K.-H. \& Huddleston D.E.: 1990, JGR 95, p 10249 • Cravens T.E., Kozyra J.U., Nagy A.F., Gombosi T.I. \& Kurtz M.: 1987, JGR 92, p 7341 - Delitsky M.L., Eviatar A. \& Richardson J.D.: 1989, GRL 16, p 215 - Desch M.D., Kaiser M.L. \& Kurth W.S.: 1989, JGR 94, p 5255 • Dubinin E.M., Lundin R., Pissarenko N.F., Barabash S.V., Zakharov A.V., Koskinen H., Schwingenschuh K. \& Yeroshenko Ye.G.: 1990, GRL 17, p 861 - Fahr H.J. \& Ziemkiewicz J.: 1988, Astron. Astrophys. 202, p 295 - Fung S.F. \& Papadopoulos K.: 1987, JGR 92, p 8579 • Gaffey J.D.,Jr, Winske D. \& Wu C.S.: 1988, JGR 93, p 5470 - Galeev A.A.: 1987, Astron. Astrophys. 187, p 12 - Galeev A.A.: 1989, GM53, p 1 - Galeev A.A. \& Sagdeev R.Z.: 1988, Astrophys. Space Sci. 144, p 427 Galeev A.A., Sagdeev R.Z., Shapiro V.D. \& Shevchenko V.I.: 1990, JGR 95 (submitted) • Gary S.P. \& Madland C.D.: 1988, JGR 93, p 235 - Gary S.P., Madland C.D., Omidi N. \& Winske D.: 1988, JGR 93, p 9584 • Gary S.P., Akimoto K. \& Winske D.: 1989, JGR 94, p 3513 • Gary S.P. \& Winske D.: 1990, JGR 95, p 8085 - Genova F. \& Calvert W.: 1988, JGR 93, p 979 - Glassmeier K.-H., Ness N.F., Acuña M.H. \& Neubauer F.M.: 1989, JGR 94, p 15063 • Goldstein M.L., Wong H.K. \& Glassmeier K.-H.: 1990, JGR 95, p 947 • Gombosi T.I.: 1988, JGR 93, p $35 \bullet$ Gosling J.T., Thomsen M.F., Bame S.J. \& Russell C.T.: 1989, JGR 94, p 3555 • Grappin R., Mangeney A. \& Marsch E.: 1990, JGR 95, p 8197 - Gurnett D.A., Kurth W.S., Granroth L., Cairns I., Macek 
W.M., Poynter R.L., Moses S.L., Coroniti F.V., Kennel C.F. \& Barbosa D.D.: 1990, COSPAR, p 23 • Hada T., Kennel C.F. \& Buti B.: 1989, JGR 94, p 65 - Hizanides K., Cargill P.J. \& Papadopoulos K.: 1988, JGR 93, p 9577 • Horton W. \& Smith R.A.: 1988, JGR 93, p 12761 • Ip W.-H.: 1990, COSPAR, p 29 - Ip W.-H. \& Banaszkiewicz M.: 1990, GRL 17, p 857 - Kaiser M.L.: 1989, GM53, p 221 - Kaiser M.L., Desch M.D. \& Curtis S.A.: 1987, JGR 92, p 15169 • Kaiser M.L., Desch M.D. \& Connerney J.E.P.: 1989, JGR 94, p 2399 • Kennel C.F.: 1988, JGR 93, p 8545 • Khabibrakhmanov I.Kh. \& Verheest F.: 1990, JGR 95, p 10449 - Kurth W.S.: 1990, COSPAR, P 29 - Kurth W.S., Barbosa D.D., Gurnett D.A. \& Scarf F.L.: 1987, JGR 92, p 15225 • Kurth W.S., Gurnett D.A. \& Scarf F.L.: 1989, JGR 94, p 6917 • Kurth W.S., Gurnett D.A. \& Desch M.D.: 1990, JGR 95, p 1103 - Lakhina G.S. \& Shukla P.K.: 1987, Astrophys. Space Sci. 139, p 275 - Lakhina G.S. \& Buti B.: 1988, Astrophys. J. 327, p 1020 - Lakhina G.S. \& Verheest F.: 1988, Astrophys. Space Sci. 143, p 329 • Le G., Russell C.T. \& Smith E.J.: 1989, JGR 94, p 3755 • Leblanc Y., Aubier M.G., Ortega-Molina A. \& Lecacheux A.: 1987, JGR 92, p 15125 - Lecacheux A. \& Ortega-Molina A.: 1987, JGR 92, p 15148 • Lee L.C.: 1989, GM59, p 239 • Lee M.A.: 1989, GM59, p 13 - Lee M.A. \& Ip W.-H.: 1987, JGR 92, p 11041 - Luhmann J.G., Russell C.T., Scarf F.L., Brace L.H. \& Knudsen W.C.: 1987, JGR 92, p 8545 • Marsch E. \& Livi S.: 1987, JGR 92, p 7263 - Marsch E. \& Tu C.-Y.: 1990, JGR 95, p 8211 • Mendis D.A., Flammer K.R., Rème H., Savaud J.A., d'Uston C., Cotin F., Cros A., Anderson K.A., Carlson C.W., Curtis D.W., Larson D.E., Lin R.P., Mitchell D.L., Korth A. \& Richter A.K.: 1989, Ann. Geophysicae 7, p 99 • Möbius E., Klecker B., Hovestadt D. \& Scholer M.: 1988, Astrophys. Space Sci. 144, p 487 - Moses S.L., Coroniti F.V., Kennel C.F. \& Scarf F.L.: 1988, JGR 93, p 1785 \& p 8483 • Neubauer F.M.: 1987, Astron. Astrophys. 187, p 73 - Neubauer F.M.: 1988, JGR 93, p 7272 • Neugebauer M., Lazarus A.J., Altwegg K., Balsiger H., Goldstein B.E., Goldstein R., Neubauer F.M., Rosenbauer H., Schwenn R., Shelley E.G. \& Ungstrup E.: 1987, Astron. Astrophys. 187, p 21 - Neugebauer M. \& Buti B.: 1990, JGR 95, p 13 • Omidi N. \& Winske D.: 1988, GRL 15, p 1303 • Onsager T.G., Thomsen M.F., Gosling J.T., Bame S.J. \& Russell C.T.: 1990, JGR 95, p 2261 - Ponce Dawson S. \& Ferro Fontán C.: 1990, Astrophys. J. 348, p 761 - Price C.P.: 1989, JGR 94, p 15001 • Price C.P. \& Wu C.S.: 1987, GRL 14, p 856 • Price C.P. \& Lee L.C.: 1988, Astrophys. J. 324, p 606 • Price C.P., Gaffey J.D.,Jr \& Dong J.Q.: 1988, JGR 93, p 837 • Richter A.K. \& Marsch E.: 1988, Ann. Geophysicae 6, p 319 - Roberts D.A., Klein L.W., Goldstein M.L. \& Matthaeus W.H.: 1987, JGR 92, p 11021 • Roberts D.A., Goldstein M.L. \& Klein L.W.: 1990, JGR 95, p 4203 • Russell C.T., Luhmann J.G., Schwingenschuh K., Riedler W. \& Yeroshenko Ye.: 1990, GRL 17, p 897 • Russell C.T., Lepping R.P. \& Smith C.W.: 1990, JGR 95, p 2273 • Sagdeev R.Z., Shapiro V.D., Shevchenko V.I., Zacharov A., Király P., Szegő K., Nagy A.F. \& Grard R.J.L.: 1990, GRL 17, p 893 • Sauer K., Motschmann U. \& Roatsch Th.: 1990, Ann. Geophysicae 8, p 243 - Scarf F.L.: 1989, GM53, p 31 - Scarf F.L., Gurnett D.A., Kurth W.S., Coroniti F.V., Kennel C.F. \& Poynter R.L.: 1987, JGR 92, p 15217 - Scholer M., Möbius E., Kistler L.M., Klecker B. \& Ipavich F.M.: 1989, GRL 16, p 571 - Schwingenschuh K., Riedler W., Lichtenegger H., Yeroshenko Ye., Sauer K., Luhmann J.G., Ong M. \& Russell C.T.: 1990, GRL 17, p 889 • Smith C.W., Goldstein M.L. \& Wong H.K.: 1989, JGR 94, p 17035 - Terasawa T. \& Scholer M.: 1989, Science 244, p 1050 • Thomas V.A. \& Brecht S.H.: 1988, JGR 93, p 11341 • Thomsen M.F., Gosling J.T., Bame S.J., Quest K.B., Russell C.T. \& Fuselier S.A.: 1988, JGR 93, p 11311 - Thomsen M.F., Gosling J.T., Bame S.J. \& Russell C.T.: 1990, JGR 95, p 957 • Thorne R.M. \& Tsurutani B.T.: 1987, Planet. Space Sci. 35, p 1501 - Tsurutani B.T., Thorne R.M., Smith E.J., Gosling J.T. \& Matsumoto H.: 1987, JGR 92, p 11074 - Tsurutani B.T., Brinca A.L., Buti B., Smith E.J., Thorne R.M. \& Matsumoto H.: 1989, JGR 94, p 29 - Tu C.-Y: 1988, JGR 93, p 7 - Tu C.-Y., Marsch E. \& Rosenbauer H.: 1990, GRL 17, p 283 - Verheest F.: 1990, Icarus 86, p 273 - Wallis M.K.: 1987, Mon. Not. R. astr. Soc. 227, p 331 - Whang Y.C.: 1988, JGR 93, p $251 \bullet \quad$ Wolfson R.: 1987, JGR 92, p 9875 - Wong H.K. \& Goldstein M.L.: 1988, JGR 93, p 4110 - Wright A.N.: 1987, JGR 92, p 9963 • Wu C.S., He X.T. \& Price C.P.: 1988, JGR 93, p 3949 • Wu C.S. \& Yoon P.H.: 1990, JGR 95, p 10273 • Yeroshenko Ye., Riedler W., Schwingenschuh K., Luhmann J.G., Ong M. \& Russell C.T.: 1990, GRL 17, p 885 - Yoon P.H.: 1990, GRL 17, p 1033 - Yumoto K., Saito T. \& Nakagawa T.: 1987, Astron. Astrophys. 187, p 117 